\title{
Evaluation of reproductive profile in male albino rats following varied duration of administration with Revive capsule
}

\author{
Onengiyeofori Ibama ${ }^{1 *}$, Kinikanwo I. Green ${ }^{2}$, Edna O. Nwachuku ${ }^{3}$, \\ Donatus O. Onwuli ${ }^{3}$, Adline Erinma Ben-Chioma ${ }^{3}$
}

\begin{abstract}
${ }^{1}$ Department of Chemical Pathology, Faculty of Basic Clinical Science, College of Medical Sciences, Rivers State University, Port-Harcourt, Nigeria

${ }^{2}$ Department of Obstetrics and Gynecology, University of Port-Harcourt Teaching Hospital, Port-Harcourt, Nigeria

${ }^{3}$ Department of Medical Laboratory Science, Faculty of Sciences, Rivers State University, Port-Harcourt, Nigeria
\end{abstract}

Received: 20 June 2021

Accepted: 19 July 2021

\author{
*Correspondence: \\ Dr. Onengiyeofori Ibama, \\ E-mail: onengs4u@yahoo.com
}

Copyright: (c) the author(s), publisher and licensee Medip Academy. This is an open-access article distributed under the terms of the Creative Commons Attribution Non-Commercial License, which permits unrestricted non-commercial use, distribution, and reproduction in any medium, provided the original work is properly cited.

\begin{abstract}
Background: Revive capsule is a polyherbal formulation commonly used to treat erectile dysfunction or enhance libido in men. Some of the individual herbs used in the formulation of this drug have been known scientifically to affect various biochemical components of the human body; hence this study was aimed at evaluating the reproductive profile in male albino rats following varied duration of administration with Revive capsule.

Methods: A total of 42 male albino rats were used for the study, and were divided into six (6) groups of seven (7) rats each. They were allowed to acclimatize for two (2) weeks by maintaining 12-hour light and dark cycles daily, with access to standard feed and water ad libitum. Group A (negative control) rats were administered with distilled water once daily, while groups B, C, D, E and F were administered once daily with $72 \mathrm{mg} / \mathrm{kg}$ of Revive capsule for 1 week, 2 weeks, 3 weeks, 4 weeks and 6 weeks respectively. The rat dose administered was extrapolated from the human dose using the formula by Paget and Barnes. At the end of each treatment week, the rats were allowed to fast overnight, followed by their anaesthetization using chloroform, and blood sample collection via jugular vein puncture. Also, the testes were excised; the epididymis were also excised from the testes and used immediately for semen analysis, while the epididymis-free testes were examined histologically. Rat-specific test kits with ELISA method were used to analyze serum LH, FSH and testosterone.

Results: The results showed a significant increase $(\mathrm{p}<0.05)$ in serum LH, FSH and testosterone levels, and a significant increase in sperm count and sperm quality parameters in the treatment groups compared to the negative control, with the maximum levels attained after 6 weeks of treatment (group F). Also, photomicrographs of histologically examined testes of the treatment groups appeared indifferent from those of the negative control.

Conclusions: These findings may suggest that in using a rat model, treatment with Revive capsule at the appropriate dosage for 6 weeks is safe, and that, besides its acclaimed use in enhancing libido or treating erectile dysfunction, it may also be effective in promoting male fertility.
\end{abstract}

Keywords: Revive, Sildenafil citrate, Phytochemicals, Erectile dysfunction, Libido, Fertility

\section{INTRODUCTION}

Revive capsule (also called Kedi Revive) is a polyherbal formulation manufactured by Kedi healthcare company limited in Hong Kong, China. It is composed of several herbs known to possess aphrodisiac properties, and is used for the treatment of erectile dysfunction and enhancement of libido in men. Its effects are exerted 4 hours after administration via the oral route. Each capsule of the drug contains $400 \mathrm{mg}$ of the constituent herbs which include; 80 $\mathrm{mg}$ of herb Epimedii (mainly Epimedium sagittatum), 80 $\mathrm{mg}$ of Radix ginseng, $40 \mathrm{mg}$ of Cordyceps militaris, $80 \mathrm{mg}$ 
of Tribulus terrestris, $80 \mathrm{mg}$ of Radix polygoni multiflori, and $40 \mathrm{mg}$ of Eucommia ulmoides. The dosage for an adult man is two capsules $(800 \mathrm{mg}$ ) to be taken once daily for a minimum of 28 days (to expect significant improvement when used to treat erectile dysfunction). Due to their mechanism of action (similar to that of Sildnafil citrate, vardenafil and Tadalafil), these drugs are referred to as phosphodiesterase type-5 inhibitors. They are mildly vasoactive in nature and exert their pharmacological effects by selectively inhibiting an enzyme called phosphodiesterase type-5 (PDE-5). This enzyme promotes the hydrolysis of cyclic guanosine monophosphate (cGMP) responsible for the relaxation of the smooth muscle in the cavernosum tissue of the penis; the cGMP is also a second messenger of nitric oxide. ${ }^{1}$ This inhibition of PDE-5 increases arterial blood flow, induces a rise in cGMP levels, and decreases intracellular calcium $\left(\mathrm{Ca}^{2+}\right)$ which enhances the smooth muscle to relax, the blood vessels to dilate and the penis to become erect. ${ }^{2}$

\section{METHODS}

The study period was from June, 2020 to February, 2021.

\section{The experimental animals}

A total of forty-two (42) male albino rats weighing between 130 to $220 \mathrm{~g}$ were used for this study. The rats were divided into six (6) groups of seven (7) rats each. Each group of the rats were put into well-labeled cages, and were allowed to acclimatize for two (2) weeks by maintaining light and dark cycles for 12 hours daily, with access to standard rat feeds and water ad libitum.

\section{The drugs used for the study}

The drug used for this study was Revive capsule. The drug is a polyherbal formulation produced by Kedi healthcare company limited, Hong Kong in China and sold in Nigeria. However, the phytochemicals in Revive capsule were determined qualitatively using a classical method, and quantitatively using a spectrophotometric method. ${ }^{3}$

\section{Acute toxicity study}

This was done to determine the LD50 of Revive capsule, using the fixed dose procedure, in which three (3) male albino rats were put together in a cage, and allowed to fast overnight, after which each of the rats was administered with Revive capsule at a dose of $2000 \mathrm{mg} / \mathrm{kg}$ body weight. ${ }^{4}$ Then, they were physically observed for 72 hours ( 3 days) for signs of toxicity or death. However, no sign of toxicity or fatality was physically recorded; this may imply that the herbal formulation Revive was safe at a single dose even at a dosage of $2000 \mathrm{mg} / \mathrm{kg}$.

\section{Calculation of doses}

The doses administered were extrapolated from the human dose using the formula by Paget and Barnes, which is represented as rat dose $=$ human $\operatorname{dosex} 0.018 \times 5.5$ The rats in each group were weighed, and the average of their weights taken. Based on the average weight taken, the appropriate dose (expressed $\mathrm{mg} / \mathrm{kg}$ body weight of the rats) of the drug for each group was determined, as well as the appropriate volume of distilled water used as diluent for the drugs following the OECD guideline; this preparation was done weekly throughout the period of the drug administration. ${ }^{4}$

Each capsule is $400 \mathrm{mg}$, and the adult human dosage is 800 mg (two capsules) to be taken once daily. Therefore, the rat dose $(\mathrm{mg} / \mathrm{kg})=$ human dose $(800 \mathrm{mg}) \times 0.018 \times 5$, which is equal to $72 \mathrm{mg} / \mathrm{kg}(72 \mathrm{mg} / 1000 \mathrm{~g})$ body weight of each rat (for a rat weighing $200 \mathrm{~g}$ ).

Group A (Negative control group) was administered with distilled water once daily.

Groups B, C, D, E and F were treated with Revive capsule at a dosage of $72 \mathrm{mg} / \mathrm{kg}$ once daily for 1 week, 2 weeks, 3 weeks, 4 weeks and 6 weeks respectively.

At the end of each treatment period, the rats were allowed to fast overnight, and were anaesthetized using chloroform. Then $5 \mathrm{ml}$ of whole blood specimen was collected (using a sterile syringe and needle) into sterile sample containers (plain bottles) through jugular vein puncture, followed by surgical removal of the testes; the epididymis was detached and used immediately for semen analysis, while the epididymis-free testes were immediately preserved in $10 \%$ formal saline for histological analysis.

The male reproductive parameters (testosterone, FSH and LH) were analyzed using rat-specific ELISA test kits manufactured at the bioassay technology laboratory in Shanghai, China. ${ }^{6}$ The epididymis was lacerated, and the semen gently pressed out into a sterile Petri dish, and was analyzed. The histological analysis of the rats' testes was done through tissue processing, and staining using the "Haematoxylin and Eosin"' staining technique.

\section{Statistical analysis}

The data generated from the analysis were expressed as Mean \pm Standard deviation, and analyzed using the statistical package for social sciences (SPSS) version 23. Comparisons of mean and standard deviation values were made for the various parameters for tests and control using the one-way ANOVA and Tukey tests. Results were considered statistically significant at $95 \%$ confidence interval $(\mathrm{p}<0.05)$.

\section{RESULTS}

\section{Results of the phytochemical analysis of Revive capsule}

Details of this are shown in Table 1. It shows the presence of the phytochemical's flavonoid, cardiac glycoside, tannins, polyphenols, alkaloids and quinones in Revive 
capsule with mean concentrations of $20.48 \pm 1.18$, $2.76 \pm 0.15, \quad 1.43 \pm 0.43, \quad 25.4 \pm 0.19, \quad 23.87 \pm 0.44$ and $0.76 \pm 0.01$ respectively.

Table 1: Results of the phytochemical analysis of Revive capsule.

\begin{tabular}{|lll|}
\hline Components & Qualitative & Quantitative \\
\hline Flavonoid & $+^{*}$ & $20.48 \pm 1.18$ \\
\hline Cardiac glycoside & + & $2.76 \pm 0.15$ \\
\hline Tannins & + & $1.43 \pm 0.43$ \\
\hline Polyphenols & ++ & $25.4 \pm 0.19$ \\
\hline Alkaloids & ++ & $23.87 \pm 0.44$ \\
\hline Quinones & + & $0.76 \pm 0.01$ \\
\hline *+: present & & \\
\hline
\end{tabular}

\section{Comparison between the mean serum levels of $\mathrm{LH}, \mathrm{FSH}$ and testosterone of the various groups}

Details of this are shown in Table 2. It shows that the mean LH level of group F was significantly higher compared to those of other groups, but no significant difference occurred between the other groups. Also, the mean LH level of group D was significantly higher compared to that of group A. The mean FSH level of group F was also significantly higher compared to those of groups B, C and $\mathrm{E}$; group $\mathrm{D}$ was significantly higher compared to groups $\mathrm{A}, \mathrm{B}, \mathrm{C}$ and $\mathrm{E}$, and group $\mathrm{C}$ was significantly higher compared to group A; however, no significant difference occurred between groups A, B and E, and no significant difference between groups $\mathrm{B}$ and $\mathrm{C}$ when compared. The mean testosterone level of group $\mathrm{F}$ was significantly higher compared to those of other groups; group E was significantly higher compared to group A and B; group D was significantly higher compared to group A, B, and C; however, no significant difference was noted between group $\mathrm{A}, \mathrm{B}$ and $\mathrm{C}$, and between group $\mathrm{C}$ and $\mathrm{E}$.

Comparison between the mean semen volume, sperm viability and normal morphology of the sperm cells of the various groups

Details of this are shown in Table 3. It shows that the mean semen volume of group $\mathrm{F}$ is significance higher compared to the mean level of group A, C and E; the mean semen volume of group $\mathrm{E}$ is significantly lower compared to that of group B; the mean level of group $\mathrm{V}$ was significantly higher compared to that of group A; the mean level of group B was significantly higher compared to that of group A. No significant difference was noted in the mean viability across all groups. However, the mean normal morphology of group $\mathrm{F}$ was significantly increased compared to that of group $\mathrm{B}$ and $\mathrm{E}$; the mean normal morphology of group E was significantly higher compared to that of group D; and group D was significantly higher compared to group B; however, no significant difference was noted between group $\mathrm{A}$ and other groups when compared.

Table 2: Results of LH, FSH and testosterone of groups A-F compared.

\begin{tabular}{|llll|}
\hline $\mathbf{N}=7$ & $\mathrm{LH}(\mathrm{mIU} / \mathrm{mL})$ & FSH $(\mathbf{m I U} / \mathbf{m L})$ & Testosterone $(\mathrm{ng} / \mathrm{L})$ \\
\hline Group A $(\mathbf{N C})$ & $0.84 \pm 0.13^{\mathrm{gi}}$ & $1.24 \pm 0.21^{\text {fgi }}$ & $95.17 \pm 1.04^{\text {ghi }}$ \\
\hline Group B & $1.08 \pm 0.17^{\mathrm{i}}$ & $1.48 \pm 0.28^{\mathrm{gi}}$ & $95.86 \pm 1.71^{\text {ghi }}$ \\
\hline Group C & $1.06 \pm 0.23^{\mathrm{i}}$ & $2.02 \pm 0.82^{\mathrm{di}}$ & $97.75 \pm 1.68^{\mathrm{gi}}$ \\
\hline Group D & $1.41 \pm 0.20^{\mathrm{di}}$ & $2.66 \pm 0.43^{\mathrm{deh}}$ & $103.34 \pm 2.35^{\text {defi }}$ \\
\hline Group E & $1.09 \pm 0.13^{\mathrm{i}}$ & $1.73 \pm 0.25^{\text {fi }}$ & $101.95 \pm 2.79^{\text {dei }}$ \\
\hline Group F & $2.43 \pm 0.59^{\text {defgh }}$ & $3.06 \pm 0.33^{\text {defh }}$ & $112.07 \pm 5.17^{\text {defgh }}$ \\
\hline F value & 27.637 & 18.045 & 35.863 \\
\hline P value & $<0.001^{*}$ & $<0.001^{*}$ & $<0.001^{*}$ \\
\hline
\end{tabular}

* = statistically significant, $\mathrm{n}=$ number of samples, ${ }^{\mathrm{d}}=$ significantly different from group $\mathrm{A}, \mathrm{e}^{\mathrm{e}}=$ significantly different from group $\mathrm{B}, \mathrm{f}_{=}$ significantly different from group $\mathrm{C},{ }^{\mathrm{g}}=$ significantly different from group $\mathrm{D},{ }^{\mathrm{h}}=$ significantly different from group $\mathrm{E},{ }^{\mathrm{i}}=\operatorname{significantly}$ different from group F.

Table 3: Results of semen volume (V), sperm viability (SV) and normal morphology of the sperm cells (NM) of groups A-F compared.

\begin{tabular}{|llll|}
\hline N=7 & Volume $(\mathrm{ml})$ & Viability $(\%)$ & Normal Morphology \\
\hline Group A $(\mathbf{N C})$ & $0.11 \pm 0.04^{\mathrm{egi}}$ & $81.43 \pm 6.27$ & $81.43 \pm 4.76$ \\
\hline Group B & $0.30 \pm 0.12^{\mathrm{dh}}$ & $80.00 \pm 11.55$ & $75.00 \pm 9.13^{\mathrm{gi}}$ \\
\hline Group C & $0.20 \pm 0.10^{\mathrm{i}}$ & $84.29 \pm 4.50$ & $80.00 \pm 4.08$ \\
\hline Group D & $0.26 \pm 0.05^{\mathrm{d}}$ & $86.43 \pm 3.78$ & $87.14 \pm 4.88^{\mathrm{eh}}$ \\
\hline Group E & $0.15 \pm 0.05^{\mathrm{ei}}$ & $83.57 \pm 3.78$ & $75.00 \pm 7.07^{\mathrm{gi}}$ \\
\hline Group F & $0.36 \pm 0.08^{\mathrm{dfh}}$ & $84.29 \pm 3.5$ & $86.43 \pm 4.76^{\text {eh }}$ \\
\hline F value & 10.057 & 0.943 & 5.377 \\
\hline P value & $<0.001^{*}$ & 0.465 & $0.001^{*}$ \\
\hline
\end{tabular}

* =statistically significant, $\mathrm{n}=$ no. of samples, ${ }^{\mathrm{d}}=$ significantly different from group $\mathrm{A},{ }^{\mathrm{e}}=$ significantly different from group $\mathrm{B},{ }^{\mathrm{f}}=$ significantly different from group $\mathrm{C},{ }^{\mathrm{g}}=$ significantly diff. from group $\mathrm{D},{ }^{\mathrm{h}}=$ significantly different from group $\mathrm{E},{ }^{\mathrm{i}}=$ significantly different from group $\mathrm{F}$ 
Table 4: Results of the mean levels of actively-motility sperm cells (AM), sluggishly-motility sperm cells (SM), nonmotile sperm cells (NM) and sperm count (SC) of the various groups compared.

\begin{tabular}{|lllll|}
\hline N=7 & AM $(\%)$ & SM $(\%)$ & NM $(\%)$ & SC $\left(\mathbf{X ~ 1 0 ^ { 6 }}\right.$ cells/mL) \\
\hline Group A & $76.43 \pm 8.52$ & $9.29 \pm 1.89$ & $14.29 \pm 7.87^{\mathrm{i}}$ & $357.14 \pm 44.99^{\mathrm{gi}}$ \\
\hline Group B & $75.00 \pm 5.77^{\mathrm{gi}}$ & $10.00 \pm 0.00^{\mathrm{g}}$ & $15.00 \pm 5.77^{\mathrm{i}}$ & $364.29 \pm 47.56^{\mathrm{gi}}$ \\
\hline Group C & $78.57 \pm 5.56$ & $8.57 \pm 2.44$ & $12.86 \pm 3.93$ & $428.57 \pm 56.70^{\mathrm{i}}$ \\
\hline Group D & $86.43 \pm 3.78^{\mathrm{eh}}$ & $5.71 \pm 1.89^{\mathrm{eh}}$ & $7.86 \pm 2.67^{\mathrm{h}}$ & $507.14 \pm 67.26^{\mathrm{de}}$ \\
\hline Group E & $73.57 \pm 10.29^{\mathrm{gi}}$ & $10.00 \pm 2.89^{\mathrm{g}}$ & $17.86 \pm 8.09^{\mathrm{gi}}$ & $421.43 \pm 69.86^{\mathrm{i}}$ \\
\hline Group F & $87.14 \pm 4.88^{\mathrm{eh}}$ & $8.57 \pm 3.78$ & $4.29 \pm 1.89^{\text {deh }}$ & $550.00 \pm 50.00^{\text {defh }}$ \\
\hline F value & 5.854 & 2.980 & 5.660 & 27.211 \\
\hline P value & $<0.001^{*}$ & $0.024^{*}$ & $0.001^{*}$ & $<0.001^{*}$ \\
\hline
\end{tabular}
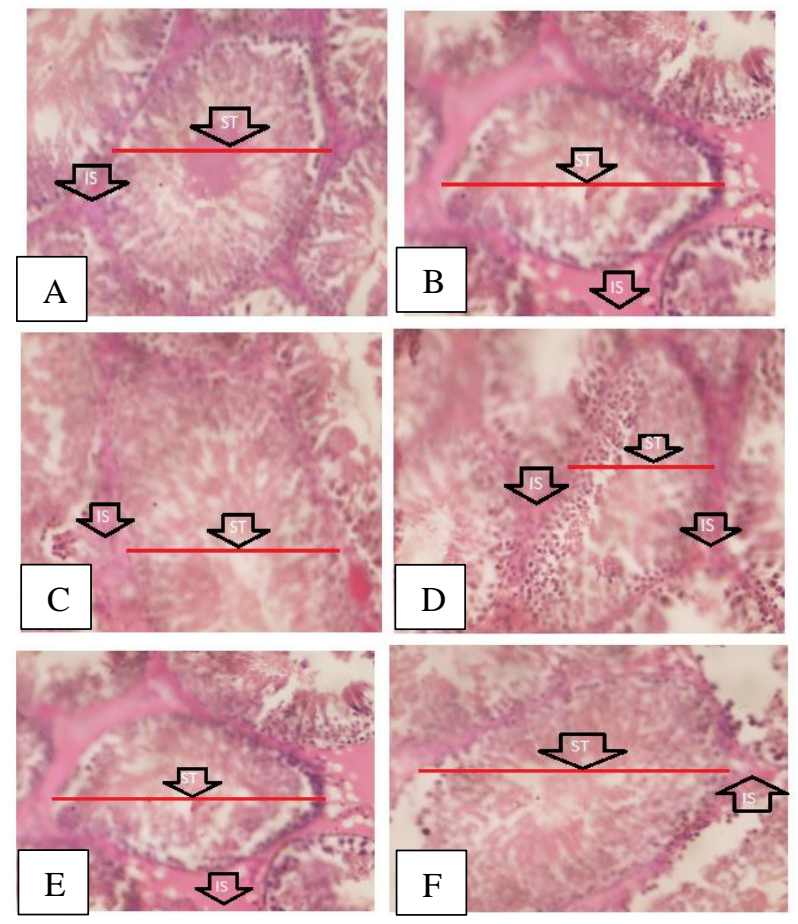

Figure 1 (A, B, C, D, E and F) Photomicrograph (X 400) $\mathrm{H}$ and $\mathrm{E}$-stained histologic sections of the testes

of the rats. The negative control (group A) shows

histologically normal testes presenting seminiferous

tubule (ST) containing spermatogenic cells and spermatozoa at the luminal border, and interstitial space (IS) containing Leydig cells. The other groups also show histologically normal testes.

Comparison between the mean levels of actively-motility sperm cells (AM), sluggishly-motility sperm cells (SM), non-motile sperm cells (NM) and sperm count (SC) of the various groups

Details of this are shown in Table 4 below. It shows that the mean AM level of group F was significantly increased when compared with that of group B and E; the mean AM level of group $\mathrm{E}$ was significantly higher than that of group D; group D was significantly elevated when compared with group $\mathrm{B}$; however, no statistically significant difference occurred between group A and other groups when compared. The mean SM level of group E was significantly lower compared to that of group D; group D was significantly lower compared to group B; however, there was no significant difference between group A and other groups. The mean NM level of group $\mathrm{F}$ was significantly lower compared to that of group A, B and E; group $\mathrm{E}$ was significantly higher compared to group $\mathrm{D}$; but no significant difference was noted between group A, B, C and D when compared. The mean SC level of group F was significantly higher compared to that of all other groups (except group D); group E was significantly higher compared to group A; group D was significantly higher compared to group A, B, C and E; finally, group A was significantly lower than all other groups.

\section{DISCUSSION}

Phytochemical analysis of the polyherbal drug Revive showed the presence of flavonoid, cardiac glycosides, tannins, phenols, alkaloids, and quinones in different quantities. Herbs or herbal products contain several bioactive phytochemicals which, apart from possessing nutritive value, may also exert pharmacological responses by influencing metabolic pathways, and are hence useful in medicine. ${ }^{8}$

Results from this study showed that the mean LH level of group F was significantly higher when compared to those of the other groups. When compared to the negative control, the mean LH value began to increase nonsignificantly after one week of treatment (group B), and even after two weeks of treatment (group C). However, a significant increase was noted after three weeks of treatment (group D), with the maximum significant increase attained after 6 weeks of treatment (group F). Similarly, the mean FSH level of group F was significantly higher when compared to those of the other groups. The mean FSH value began to increase non-significantly after one week of treatment (group B) when compared to the negative control. However, a significant increase was noted after two weeks of treatment (group C), with the maximum significant increase attained after 6 weeks of treatment (group F). From these results, the serum levels of LH and FSH increased as the duration of treatment with Revive capsule increased, with the maximum increase noted after 6 weeks of treatment. The increased serum LH and FSH levels noted from this study may be attributed to the phytochemicals present in the drug; Revive capsule being a polyherbal drug, is made up of several medicinal 
herbs including Epimedium sagittatum (also called Herba epimedii), this plant contains several active components, with the major component being the flavonoids (particularly icariin). ${ }^{9}$ A study conducted by Zhang and Yang reported an elevated serum LH and FSH levels in male rats treated with icarrin. ${ }^{10}$ The reports from this study may also be attributed to the individual herbs (containing phytochemicals) used in the formulation of the Revive capsule; Munir et al conducted a study by assessing the therapeutic response of different doses of Epimedium gandiflorum (an herb present in Revive capsule); they reported that oral administration of different doses of the plant's extract induced an increase in serum LH and FSH levels in albino rats pretreated with carbon-tetrachloride. ${ }^{11}$

The mean testosterone level of group $\mathrm{F}$ was significantly higher when compared to those of the other groups. There was a non-significant increase in groups B (after one week of treatment) and C (after two weeks of treatment) when compared with the negative control (group A). However, a significant increase was noted in groups D, E and F (after 3,4 , and 6 weeks of treatment). Also, this report may be attributed to the phytochemicals present in the Revive capsule; Zhang and Yang conducted a study in which male rats were treated with icarrin (a flavonoid), with a report of an increase in the serum testosterone levels in rats, and that icarrin possesses the potential to mimic testosterone, and can therefore be used to manage hypoandrogenism. ${ }^{10}$ Several herbs present in the Revive capsule have been reported scientifically to increased serum testosterone; Wang et al carried out a study by treating albino rats with bisphenol A, which induced a decreased serum LH and testosterone levels. ${ }^{12}$ The rats were then treated with Cordyceps militaris extract (containing mainly flavonoids), which in turn, induced an increase in the serum LH and testosterone levels. Munir et al also reported an elevated serum testosterone level as a result of orally administrating different doses of the extract of the herb Epimedium gandiflorum. ${ }^{11}$ The reports obtained from this study conforms with that of Boepple et al, stating that, an increase in LH and FSH secreted from the anterior pituitary in response to $\mathrm{GnRH}$ (secreted from the hypothalamus), results in an increase in the gonadal hormone, testosterone secreted by Leydig cells in testes. ${ }^{13}$

There was a significant increase in the mean semen volume after a week of treatment (group B), 3 weeks of treatment (group D) and 6 weeks treatment (group F) when compared to the negative control. When compared to other groups, group $\mathrm{F}$ had the highest significant increase in semen volume. About $70 \%$ of the normal ejaculate volume is contributed by the seminal vesicles. The report from this study may be due a stimulatory action of the Revive capsule on the vas deferens which secretes the semen.

There was no significant difference in the mean sperm viability when all the groups were compared. Sperm viability refers to the functional capacity of sperm cells, that is, alive or dead. The report from this study may suggest that the drug did not pose any effect on the viability of the sperm cells. Similarly, there was no significant difference in the mean normal morphology of the sperm cells when all the treatment groups were compared with the negative control, however, there was a non-significant decrease in group B (after 1 week of treatment) compared with the negative control, and a nonsignificant increase in group C (after 2 weeks of treatment) compared with group B. There was also, a significant increase in group D ( 3 weeks of treatment) and F (6 weeks of treatment) compared to group B. The mean levels of sperm cells with normal morphology later decreased in group E (after 4 weeks of treatment) compared to group D, and then significant increase in group F compared to group E. From this report, since none of the treatment groups had a significant increase or decrease when compared to the negative control, it may imply that drug did not pose any significant effect on normal morphology of sperm cells.

There was no significant difference in the mean levels of the actively motile (AM) sperm cells in all the treatment groups when compared to the negative control, however, group D (after 3 weeks of treatment) and F (after 6 weeks of treatment) were significantly higher when compared to group B (after 1 week of treatment). The level significantly decreased in group E (after 4 weeks of treatment) compared to group D, and was later significantly increased in group $\mathrm{F}$ when compared with group E. From this report, since none of the treatment groups had a significant increase or decrease when compared to the negative control, it may imply that the drug did not pose any significant effect on the active motility of the sperm cells. Similarly, there was no significant difference in the mean levels of the sluggishly motile (SM) sperm cells in all the treatment groups when compared to the negative control, however, there was a significant decrease in group D (after 3 weeks of treatment) compared to group B (after 1 week of treatment), and then becomes significantly increased in group E (after 4 weeks of treatment) compared to group D. For the mean level of non-motile (NM) sperm cells, there was a significant decrease in group F (after 6 weeks of treatment) compared to groups A (negative control), B and E. Sperm motility is one of the most essential parameters used to evaluate the fertility potential of a semen specimen. A motile sperm (whether actively or sluggishly) swimming straight (and not in circles) can penetrate the cervical mucus to fertilize an ovum, whereas, a non-motile sperm (whether viable or not) cannot penetrate the cervical mucus to fertilize an ovum. ${ }^{14}$ The report from this study suggests that the treatment with Revive capsule decreased the percentage of non-motile sperm cells particularly after 6 weeks of treatment, which invariably decreased the possibility of infertility.

The mean level of the sperm count (SC) after 6 weeks of treatment (group F) was significantly higher when compared to those of groups A (negative group), B, C and E. There was also a significant increase in group $D$ compared to groups A and B. After 4 weeks of treatment (group E) the mean level of SC significantly decreased when compared to that treated for 3 weeks (group D), and 
then significantly increased after 6 weeks of treatment, which happens to be the maximum increase. The report from this study may be attributed to the phytochemicals present in the Revive capsule. Additionally, some of herbs used in the formulation of the drug have been scientifically proven to possess spermatogenic effect; for example, the spermatogenic effect of cordyceps militaris (a component of Revive capsule) supplement in Sprague-Dawley rats was investigated, and reported that the supplement induced an increase in the number of sperm cells in the rats treated with the supplement. ${ }^{15}$

The limitation of this study is that, it was conducted using an animal model, and as such cannot be conclusively inferred to humans.

\section{CONCLUSION}

This study evaluated some reproductive profile in albino rats following varied duration of administration with Revive capsule. Treatment with Revive capsule for the different durations gave rise to different effects, with the maximum effect often attained after 6 weeks of treatment; the drug induced an increase in the serum levels of gonadotrophic hormones ( $\mathrm{LH}$ and $\mathrm{FSH}$ ) and gonadal hormone (testosterone), and also increased sperm quality and count, with no tissue distortion in the testes as revealed by photomicrographs of the histologically examined testes. Thus, the drug may be said to have possessed the potential of stimulating or modulating the physiology of the hypothalamic-pituitary-gonadal (HPG) axis, which may consequently promote male fertility. Therefore, after 6 weeks of treatment with Revive capsule, no pathological effect was observed or recorded in the albino rats, as such the drug is considered safe when administered at the appropriate dosage for a period of 6 weeks, and may be used to promote male fertility.

\section{ACKNOWLEDGMENTS}

Author would like to thanks who played a role in ensuring the success of this study.

\section{Funding: No funding sources}

Conflict of interest: None declared

Ethical approval: The study was approved by the Institutional Ethics Committee

\section{REFERENCES}

1. Sesti C, Florio V, Johnson EG, Kloner RA. The phosphodiesterase-5 inhibitor tadalafil reduces myocardial infarct size. Int $\mathrm{J}$ Impotence Res. 2007;19(1):55-61.

2. Aversa A, Pili M, Fabbri A, Spera E, Spera G. Erectile dysfunction: expectations beyond phosphodiesterase type-5 inhibition. J Endocrinol Investment. 2007;27(1):192-206.

3. Erel OA. Novel automated direct measurement method for total antioxidant capacity using a new generation, more stable ABTS radical cation. Clin Biochem. 2004;37(1):277-85.

4. Organization for Economic Cooperation and Development. Guidance Document on Acute Oral Toxicity Testing. 2001. Available at: https://ntp.niehs.nih.gov/iccvam/suppdocs/feddocs/oecd /oecd-gd24.pdf. Accessed on $23^{\text {rd }}$ November, 2020.

5. Paget GE, Barnes JM. Evaluation of drug activities. In Lawrence, D. R. and Bacharach, A. L. (Eds.). Pharmacometrics. New York: Academy Press. 1964;161.

6. Ibama O, Chioma U, Konne JL, Konne F. Effect of Ingested Foods Preheated (With Microwave) in Plastic Containers on the Reproductive Profile of Male Albino Rats. J Adv Med Med Res. 2020;32(24):71-5.

7. Ibama O, Konne FE. Oncogenicity of Tobacco-Smoking: A Review on Squamous Cell Carcinoma of the Tongue, Diagnosis and Treatment. World J Pharmaceutical Life Sci. 2018;4(12):1-6.

8. Kaur R, Afzal M, Kazmi I, Ahamd I, Ahmed Z, Ali B et al. Polypharmacy (Herbal and synthetic drug combination): a novel approach in the treatment of type2 diabetes and its complications in rats. J Natural Med. 2013;67(3):662-71.

9. Wu H, Lien EJ, Lien LL. Chemical and pharmacological investigations of Epimedium species: a survey. Progress Drug Res. 2003;60(1):1-57.

10. Zhang ZB, Yang QT. The testosterone mimetic properties of icariin. Asian J Androl. 2006;8(5):601-5.

11. Munir N, Mahmood Z, Yameen M, Mustafa G. Therapeutic Response of Epimedium gandiflorum's Different Doses to Restore the Antioxidant Potential and Reproductive Hormones in Male Albino Rats. DoseResponse: An Int J. 2020;1-13.

12. Wang J, Chena C, Jiang Z, Wang M, Jiang H, Zhang X. Protective effect of Cordyceps militaris extract against bisphenol A induced reproductive damage. Systems Biol Reproductive Med. 2016;62(4):249-57.

13. Boepple PA, Hayes FJ, Dwyer AA, Raivio T, Lee H, Crowley WF. Relative roles of inhibin B and sex steroids in the negative feedback regulation of follicle-stimulating hormone in men across the full spectrum of seminiferous epithelium function. J Clin Endocrinol Metabolism. 2008;93(5):1809-14.

14. Baker AM, Roberts TM, Stewart M. 2.6 A resolution crystal structure of helices of the motile major sperm protein (MSP) of Caenorhabditis elegans. J Molecular Biol. 2002;319(1):491-99.

15. Chang Y, Jeng K-C, Huang K-F, Lee Y-C, Hou C-W, Chen K-H et al. Effect of Cordyceps militaris Supplementation on Sperm Production, Sperm Motility and Hormones in Sprague-Dawley Rats. Am J Chinese Med. 2008;36(5):849-59.

Cite this article as: Ibama O, Green KI, Nwachuku EO, Onwuli DO, Ben-Chioma AE. Evaluation of reproductive profile in male albino rats following varied duration of administration with Revive capsule. Int $\mathbf{J}$ Reprod Contracept Obstet Gynecol 2021;10:2981-6. 